\title{
Coreless vortex ground state of the rotating spinor condensate
}

\author{
J.-P. Martikainen ${ }^{1-3}$, A. Collin ${ }^{2}$, and K.-A. Suominen ${ }^{1,2}$ \\ ${ }^{1}$ Department of Physics, University of Turku, FIN-20014 Turun yliopisto, Finland \\ ${ }^{2}$ Helsinki Institute of Physics, PL 64, FIN-00014 Helsingin yliopisto, Finland \\ ${ }^{3}$ Institute for Theoretical Physics, Utrecht University, Leuvenlaan 4, 3584 CE Utrecht, The Netherlands
}

(October 30, 2018)

\begin{abstract}
We study the ground state of the rotating spinor condensate and show that for slow rotation the ground state of the ferromagnetic spinor condensate is a coreless vortex. While coreless vortex is not topologically stable, we show that there is an energetic threshold for the creation of a coreless vortex. This threshold corresponds to a critical rotation frequency that vanishes as the system size increases. Also, we demonstrate the dramatically different behavior of the spinor condensate with anti-ferromagnetic interactions. For anti-ferromagnetic spinor condensate the angular momentum as a function of rotation frequency exhibits the familiar discrete staircase behavior, but in contrast to an ordinary condensate the first step is to the state with angular momentum $1 / 2$ per particle.

03.75.Fi, 32.80.Pj, 03.65.-w
\end{abstract}

\section{INTRODUCTION}

The quantization of circulation for a Bose-Einstein condensate (BEC) is well known [1]. This quantization implies a crucial role for quantized vortices as carriers of angular momenta. If the condensate rotates with angular frequency $\Omega$, the ground state of the system is a configuration of quantized vortices and the number of vortices increases with the rotation frequency [2 [6]. Energetically a state with a single vortex becomes favored only after $\Omega$ exceeds certain threshold $\Omega_{c}$ that depends on the system parameters in a fairly complicated manner [7].

While these properties are well studied, the creation of spinor condensates [8.9] has added an important extra twist. In a spinor (or vectorial) condensate with spin $S$ the spin-degree of freedom is not frozen and the condensate order parameter is a vector $\zeta$ with $2 S+1$ components. This freedom with the spin-degree has some intriguing consequences. For example, in inhomogeneous magnetic field this vectorial nature of the order parameter can even imply vortex ground states [10]. Crucially for our purposes, such circulation of an order parameter does not have to be quantized [11]. If circulation is not quantized angular momenta of the system does not have to be carried by quantized vortices.

In this paper we address the question of the ground state of the rotating spinor condensate. We show that the ground state of the ferromagnetic spinor condensate at small rotation frequencies is a coreless (non-singular) vortex and we analyze its properties. Coreless vortices have also been studied by Mizushima et al. [12] who investigated rotating spinor condensates with a fixed magnetization.We mainly concentrate on the pure rotational ground state with no external magnetic field. Furthermore, we study the rotating spinor condensate with polar (anti-ferromagnetic) interactions and show how significantly the behavior of the system changes when only the sign of the antisymmetric interaction parameter is altered. In both cases there is a critical rotation fre- quency above which the ground state carries non-zero angular momentum. However, in the ferromagnetic case the amount of angular momentum can be infinitesimal whereas the corresponding polar condensate has a quantized angular momentum (in trap units) $1 / 2$ per particle. The vortex structure of a polar spinor BEC has been previously studied by Yip 13 focusing for a single constant rotation frequency.

\section{MODEL}

The Hamiltonian for the three-dimensional spin- 1 condensate in a frame rotating with frequency $\Omega$ is [12, 14,16$]$

$$
\begin{aligned}
H & =\int d^{3} \mathbf{r}\left(\frac{\hbar^{2}}{2 m} \nabla \Psi_{i}^{*} \cdot \nabla \Psi_{i}+\frac{m}{2}\left(\omega_{t r}^{2} r^{2}+\omega_{z}^{2} z^{2}\right) \Psi_{i}^{*} \Psi_{i}\right. \\
& -\Omega \Psi_{i}^{*} \hat{L} \Psi_{i}+\frac{\lambda_{s}}{2} \Psi_{i}^{*} \Psi_{j}^{*} \Psi_{j} \Psi_{i} \\
& \left.+\frac{\lambda_{a}}{2} \sum_{\alpha} \Psi_{i}^{*} \Psi_{j}^{*}\left(F_{\alpha}\right)_{i k} \cdot\left(F_{\alpha}\right)_{j k} \Psi_{k} \Psi_{l}\right)
\end{aligned}
$$

where $m$ is the atomic mass, $\omega_{t r}$ and $\omega_{z}$ are the trapping frequencies, $\hat{L}$ is the angular momentum operator, and $\mathbf{F}_{\alpha}$ is the angular momentum matrix. The parameters $\lambda_{s}$ and $\lambda_{a}$ are system specific parameters that depend on the two different scattering lengths $a_{2}$ and $a_{0}$ : $\lambda_{s}=\frac{4 \pi \hbar^{2}\left(a_{0}+2 a_{2}\right)}{3 m}$ and $\lambda_{a}=\frac{4 \pi \hbar^{2}\left(a_{2}-a_{0}\right)}{3 m}$. In the mean field limit our aim is to find the fields $\Psi_{i}(\mathbf{r})=\psi_{i}(\mathbf{r})$ that minimize this Hamiltonian. Minimization leads to the three-component Gross-Pitaevskii equations [15. We choose $\omega_{z}=(2 \pi) 1480 \mathrm{~Hz}$ and $\omega_{t r}=(2 \pi) 62 \mathrm{~Hz}$. When the trap is filled with $1.7 \times 10^{4} \mathrm{Rb}^{87}$ atoms the condition $\mu<\hbar \omega_{z}$ is satisfied. Consequently, in $z$-direction we assume the system to be in the ground state of a parabolic trap with no dynamics and our numerical calculation becomes effectively two-dimensional. This assumption scales the scattering lengths by the constant 
$\eta=\sqrt{\frac{\omega_{z}}{2 \pi \omega_{t r}}}$. For convenience we choose the unit of length as $L_{0}=\sqrt{\frac{\hbar}{m \omega_{t r}}}$ and the unit of time as $\tau_{0}=1 / \omega_{t r}$.

It is well known that the ground state structure $\psi=$ $\left(\psi_{1}, \psi_{0}, \psi_{-1}\right)^{T}=\sqrt{n} \zeta$ depends on the sign of the $\lambda_{a}$ [14. In the absence of a magnetic field, if $\lambda_{a}>0$ the energy is minimized (with a suitable choice of axis) with a nonmagnetized spinor $\zeta=(0,1,0)^{T}$. This state is referred to as the polar state. If $\lambda_{a}<0$, a fully magnetized ferromagnetic state $\zeta=(1,0,0)^{T}$ is favored. In the absence of external magnetic field we can freely rotate the order parameter (globally) without any physical changes. In particular we can rotate the ferromagnetic ground state and get an identical order parameter expressed in terms of the Euler angles $(\alpha, \beta, \tau)$ of the rotation

$$
\psi=\sqrt{n} e^{-i \tau}\left(\begin{array}{c}
e^{-i \alpha} \cos ^{2} \beta / 2 \\
\sqrt{2} \cos \beta / 2 \sin \beta / 2 \\
e^{i \alpha} \sin ^{2} \beta / 2
\end{array}\right) .
$$

The superfluid velocity is defined by $\mathbf{v}_{\mathbf{s}}=-i \frac{\hbar}{m} \zeta^{\dagger} \nabla \zeta$. If we make the rotation local and choose $\tau=-\alpha=\phi$, with $\phi=\tan ^{-1}(x / y)$, the velocity field of the condensate is given by $\mathbf{v}_{\mathbf{s}}=\frac{1}{r}(1-\cos \beta) \hat{\phi}$ 14. If $\beta(r)=0$ the velocity field vanishes, but as $\beta(r)$ increases the velocity field starts to resemble that of an ordinary singular vortex. Such a structure is called a coreless vortex which was first predicted by two studies for superfluid ${ }^{3} \mathrm{He}$. For the Mermin-Ho (MH) 11 vortex the bending angle $\beta$ must be $\frac{\pi}{2}$ at the boundary of the condensate and for the Anderson-Toulouse (AT) [17] vortex $\beta$ must be $\pi$. For the $\mathrm{MH}$ vortex in liquid ${ }^{3} \mathrm{He}$ the boundary value is imposed by the walls of the container [18].

\section{FERROMAGNETIC GROUND STATE}

The angular momentum per particle in a condensate with a coreless vortex can be anything between 0 and 2 . Also, a coreless vortex can be continuously transformed into a state with vanishing angular momentum. This happens by simply transforming the function $\beta(r)$ into zero. Due to these reasons it seems plausible that when $\lambda_{a}<0$, a coreless vortex might play a role as a carrier of angular momenta at small values of $\Omega$. We have verified this reasonable conjecture by solving the relevant twodimensional GP equations numerically for $1.7 \times 10^{4} \mathrm{Rb}^{87}$ atoms without any additional assumptions:

$$
\begin{aligned}
i \hbar \frac{\partial \psi_{-1}}{\partial t}= & \mathcal{L} \psi_{-1}+\lambda_{a}\left(\psi_{0}^{2} \psi_{1}^{*}+\left|\psi_{-1}\right|^{2} \psi_{-1}+\left|\psi_{0}\right|^{2} \psi_{-1}\right. \\
& \left.-\left|\psi_{1}\right|^{2} \psi_{-1}\right) \\
i \hbar \frac{\partial \psi_{0}}{\partial t}= & \mathcal{L} \psi_{0}+\lambda_{a}\left(2 \psi_{1} \psi_{-1} \psi_{0}^{*}+\left|\psi_{-1}\right|^{2} \psi_{0}+\left|\psi_{1}\right|^{2} \psi_{0}\right) \\
i \hbar \frac{\partial \psi_{1}}{\partial t}= & \mathcal{L} \psi_{1}+\lambda_{a}\left(\psi_{0}^{2} \psi_{-1}^{*}+\left|\psi_{1}\right|^{2} \psi_{1}+\left|\psi_{0}\right|^{2} \psi_{1}\right. \\
& \left.-\left|\psi_{-1}\right|^{2} \psi_{1}\right)
\end{aligned}
$$

$\mathcal{L}=-\frac{\hbar^{2}}{2 m} \nabla^{2}+V_{\text {trap }}-\Omega \hat{L}+\lambda_{s}\left(\left|\psi_{-1}\right|^{2}+\left|\psi_{0}\right|^{2}+\left|\psi_{1}\right|^{2}\right)$. The lowest energy configuration at small values of $\Omega$ is a coreless vortex (see an example of the superfluid velocity field in Fig. 11). A priori, there is no reason to expect a critical rotation frequency for the existence of a coreless vortex. Nevertheless, by solving the GP equations we saw that a critical frequency $\tilde{\Omega}_{c} \approx 0.06$ had to be exceeded before a finite sized coreless vortex became energetically favored. Below this critical frequency energy was minimized with the function $\beta(r)$ being zero everywhere.

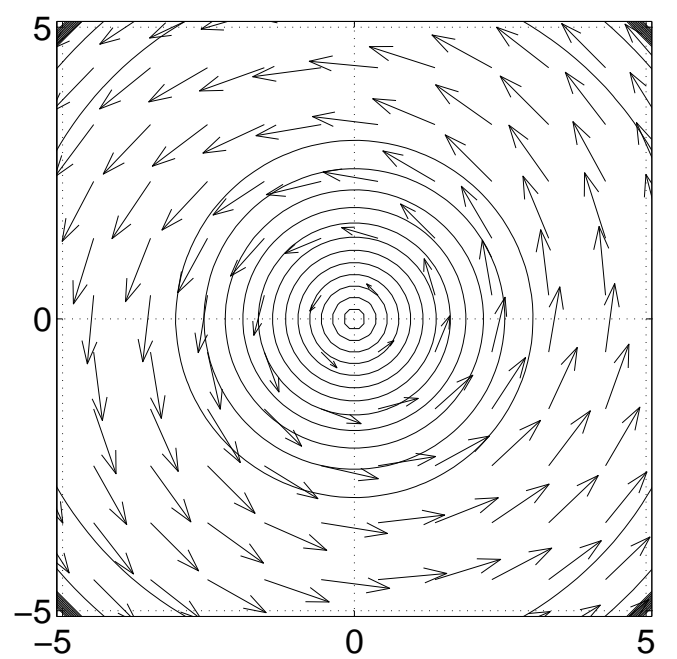

FIG. 1. Superfluid velocity field inside a rotating ferromagnetic spinor condensate for the rotation frequency $\tilde{\Omega}=0.09$. The angular momentum per particle is $L / N \approx 0.6$.

Assuming the existence of a coreless vortex the Hamiltonian in trap units is

$$
\begin{gathered}
H_{C V}[n, \beta]=\int d^{2} r n\left[\frac{1}{2} r^{2}+\frac{\lambda_{s}+\lambda_{a}}{2} n+\frac{1}{2 n}|\nabla \sqrt{n}|^{2}\right. \\
\left.+\frac{1}{4}|\nabla \beta|^{2}+\frac{3+\cos ^{2} \beta-4 \cos \beta}{4 r^{2}}-2 \tilde{\Omega} \sin ^{2} \beta / 2\right],
\end{gathered}
$$

where $\tilde{\Omega}=\Omega / \omega_{t r}$. When the rotation frequency is low and the density is large, it is reasonable to assume a density distribution almost independent of the function $\beta$ and that this density distribution has the familiar inverted parabola shape of the Thomas-Fermi approximation [19]. The $\beta(r)$ minimizing the energy functional (4) must satisfy the following differential equation

$$
\begin{aligned}
\frac{\partial^{2} \beta}{\partial r^{2}}= & -\frac{1}{r} \frac{\partial \beta}{\partial r}-\frac{\frac{\partial \beta}{\partial r} \frac{\partial n}{\partial r}}{n}+\frac{4 \sin \beta-\sin 2 \beta}{2 r^{2}} \\
& -2 \tilde{\Omega} \sin \beta .
\end{aligned}
$$

This equation is solved numerically to get the lowest energy configuration. Some results are shown in Figure 2. 
By using the same parameters as in the numerical simulations the energetical studies give $\tilde{\Omega}_{c}=0.058$ for the critical rotating frequency. This result is extremely close to the corresponding value from the GP equations. Also, the values of angular momenta as a function of $\tilde{\Omega}$ are nearly identical as can be seen in Figure 3 .

To gain more insight, Eq. (4) can also be studied variationally. With a trial $\beta(r)=r / L$ the resulting integrals can be solved. While the resulting expression is quite complicated, it is remarkable that such a simple variational model gives results that are qualitatively the same as the results based on the Eq. (5). It turns out that the the existence of an energy minimum depends (in trap units) on the magnitude of the number $A=R_{T F}^{2} \tilde{\Omega}$. Energy mimimum only exists if $A>3.05 \pm 0.05$. Thus the critical frequency predicted by the variational model with the parameters used before is $\Omega_{c}=0.064 \pm 0.001$, i.e. very close to the value calculated with more accurate methods. As the critical frequency is inversely proportional to the square of the Thomas-Fermi radius we see that the non-zero critical rotation frequency is a finite size effect. This property is also confirmed by the numerical solutions of the GP equations.

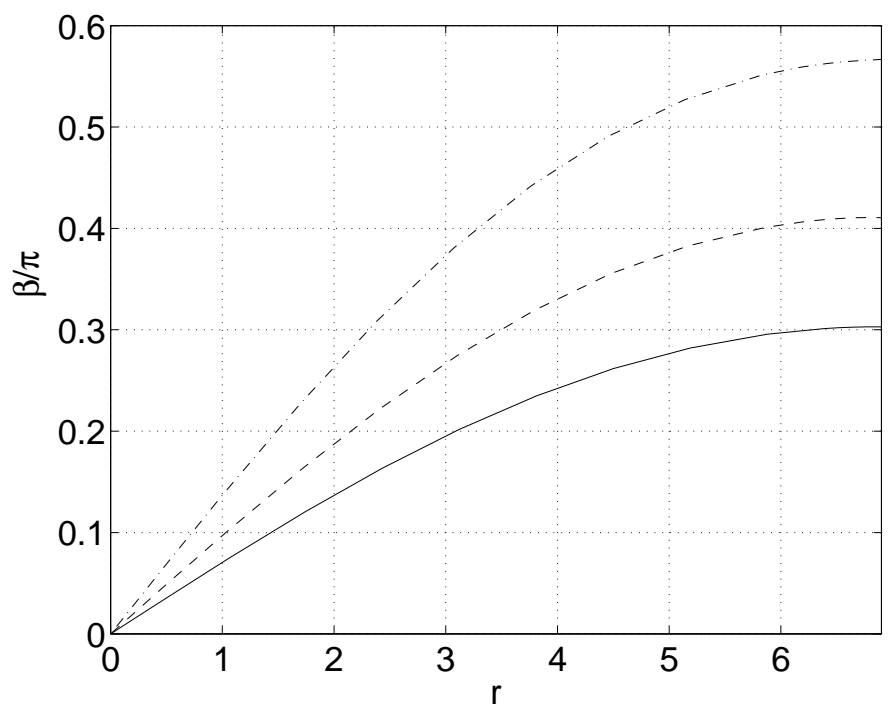

FIG. 2. The bending angle $\beta(r)$ for three different rotating frequencies: $\tilde{\Omega}=0.07$ (solid line), $\tilde{\Omega}=0.08$ (dashed line) and $\tilde{\Omega}=0.10$ (dashdotted line). The corresponding values for angular momenta are $L / N=0.24, L / N=0.42, L / N=0.74$. In this case the Thomas-Fermi radius is $r=6.9$. In order to prevent the divergence of $\beta$ at this point the second term in the right hand side of Eq. (5) is put to zero at $r=0.99 R_{T F}$.

\section{A. Ground state as a function of $\tilde{\Omega}$}

As the rotation frequency is increased, the slope of $\beta$ increases initially (to a good accuracy) linearly with the rotation frequency. This can be easily understood

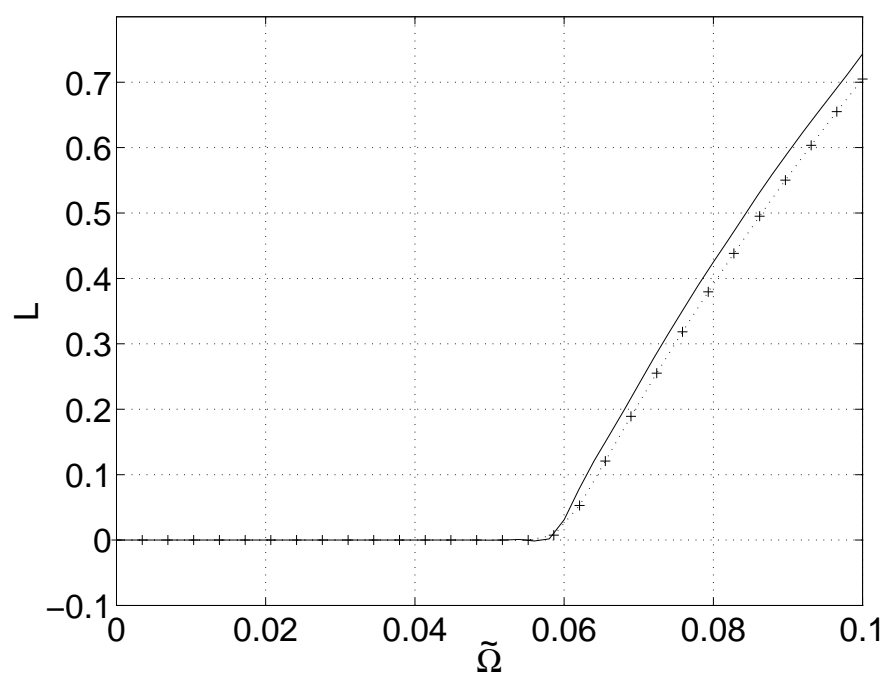

FIG. 3. The angular momentum of a ferromagnetic spin-1 condensate obtained by solving the GP equation (dotted line with plus signs) and by solving the differential equation (5) (solid line).

since initially a larger slope implies larger populations for the state with the highest winding number, namely $m=-1$ state. But if the slope becomes too large $\beta(r)$ will be larger than $\pi$ inside the condensate. If this happens, populations at states with lower winding numbers, namely $m=1$ and $m=0$ states, start to increase. This implies that angular momentum will decrease. Physically this is nonsense since it is clear that angular momentum per particle must increase monotonically with the rotation frequency. Consequently we expect an upper critical frequency $\tilde{\Omega}_{u p}$ when the coreless vortex ceases to be the ground state of the system.

We can get a rough estimate for this upper limit by assuming that the slope depends linearly on $\tilde{\Omega}$ and extrapolating the behavior to higher rotation frequencies. For the parameters used before this gives us an upper critical frequency $\tilde{\Omega}_{u p} \approx 0.15$, which is again in good agreement with the solutions of the GP equations [20].

The ground state at higher rotation frequency is more complicated. The superfluid velocity field slightly above the upper critical rotation frequency is still rotationally symmetric, but the order parameter is no longer a simple mixture of components with winding numbers 0,1 , and 2 . In Figure 1 we show an example of the order parameter when $\tilde{\Omega}=0.17$. In the $m=0$ component we can see a regular array of 4 vortices. The $m= \pm 1$ components fill the vortex cores. Although it is not clear from the figure, the $m= \pm 1$ components have a nonzero angular momenta. The velocity field of this configuration is rotationally symmetric and qualitatively resembles that in Figure 1 .

In reality $\beta(r)$ is not linear, but its slope vanishes close to the edge of the condensate. It seems that the maximum boundary value for the bending angle is $\beta_{\max }=\frac{3}{4} \pi$ 
FIG. 4. Ground state structure of the ferromagnetic spinor condensate when $\tilde{\Omega}=0.17$. The parameters used to create these figures are the same as for Figure 1. In the top most figure we show the density of the $m=1$ component, in the middle the density of the $m=0$ component, and in the lowest the density of the $m=-1$ component. Light color indicates high density. Total angular momentum per particle of this configuration is about 1.85 .

and AT-vortex is never the ground state of the system. We believe that this, somewhat surprising, boundary value is caused by a subtle effect due to kinetic energy. Ordinarily kinetic energy of the BEC can be safely ignored, but close to the boundary of the condensate where gradients are large its effects are pronounced. We conjecture that the coreless vortex structure with $\beta_{\max }>\frac{3}{4} \pi$ is dynamically unstable and that this instability is due to the physics close to the condensate edge. But we freely admit that at the time of writing this paper, a detailed understanding of the spinor condensate dynamics close to the boundary of the condensate is still lacking.

\section{ANTIFERROMAGNETIC GROUND STATE}

It should be understood that the results change dramatically with the sign of $\lambda_{a}$ [13]. While a coreless vortex can carry an infinitesimal amount of angular momentum, this state is not energetically favored if $\lambda_{a}>0$. For polar condensates the ordinary non-rotating ground state $\zeta=(0,1,0)^{T}$ remains the ground state until a certain critical frequency is reached. This critical frequency is somewhat higher than the corresponding critical frequency for a (ferromagnetic) coreless vortex, but well below the critical frequency for the stabilization of an ordinary singly quantized vortex.

Just above this threshold the ground state of the system is a state with angular momentum $1 / 2$ per particle (see Figure 5). This state is locally magnetized and cannot be represented as a local rotation of the non-rotating ground state. This is easily understood, since there is no such local rotation of the polar state that results in a texture having non-zero angular momenta. While the state is locally magnetized, the number of atoms at $m= \pm 1$ states is still the same and the system is globally non-magnetized. We can choose a convenient basis by a global rotation of the numerically calculated wavefunction. Such a procedure reveals that this state can be intepreted as a state where population is evenly split between $m= \pm 1$ states. Another one of these states carries a vortex and the other one fills the vortex core. Incidentally, the dynamical stability of this structure was predicted in Ref. 21. Here we have shown that within a certain range of rotation frequencies, this state is also the minimum energy configuration. Very interesting point is that the winding number $1 / 2$ vortex of the polar spinor condensate can viewed as the BEC counterpart to the
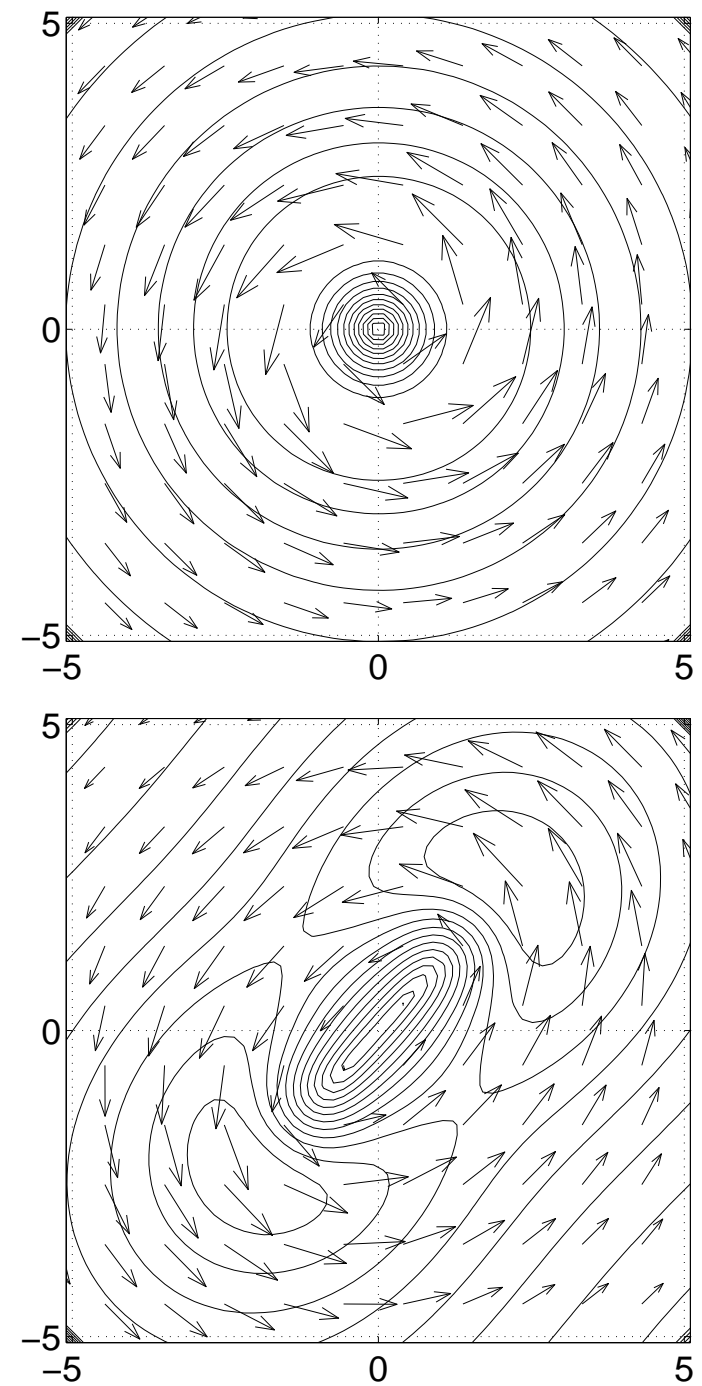

FIG. 5. The superfluid velocity field inside a rotating spinor condensate for two different rotation frequencies. Scattering lengths are such that the non-magnetic (polar) structure is favored and Thomas-Fermi radius is $R_{T F} \approx 6.9$. In the upper figure the rotation frequency is $\tilde{\Omega}=0.09$ and angular momentum per particle is $1 / 2$. In the lower figure $\tilde{\Omega}=0.1$ and angular momentum is close to 0.8 .

Alice string familiar from the particle physics [22.

Even though the behavior of the system depends strongly on the sign of $\lambda_{a}$, it does not depend strongly on its magnitude. $\lambda_{a}$ could be increased threefold without large qualitative or quantitative changes. Also, the number of particles could be changed by, at least, a factor of two without important qualitative changes. The range of rotation frequencies when this state is the ground state is quite narrow. In our simulations this range was only on the order of 0.01 . If the rotation frequency is beoynd this narrow range, the ground state is a state where population is evenly split between $m= \pm 1$ states, but in this new state both components carry a vortex. These vortices are not overlapping and the total angular mo- 
mentum per particle is less than 1. These results are analogous to the results for scalar condensates, where it has been shown that in a parabolic trap a multiply quantized vortex is never the ground state [4]6]. In a parabolic trap it is always energetically favorable to break a multiply quantized vortex into pairs of singly quantized vortices.

\section{MAGNETIC FIELD}

So far we have assumed that the chemical potentials of the different $m$-states are equal. In this section we discuss the effect of an external magnetic field pointing along the rotation axis. If the magnetic field has components also in $x$ - and $y$-directions, things can be different, as will be discussed in the next section.

External magnetic field will shift the chemical potential of the component $m$ into $\mu_{m}=\mu_{0}+m E_{Z}$, where $E_{Z}$ is the Zeeman shift and $\mu_{0}$ is the chemical potential of the $m=0$ component. An external magnetic field will tend to magnetize the system and move more population into the state with the lowest chemical potential. It is simple to show, that for a non-rotating homogeneous spinor condensate the Zeeman shift required for a complete magnetization in the direction of the external magnetic field is on the order of $\lambda_{a} n$, where $n$ is the density [23]. Note that the magnetizing field is very small e.g. for sodium atoms with reasonable densities it is about $B=10^{-4}-10^{-3} \mathrm{G}$.

Assume a slowly rotating system prepared in the absence of the magnetic field and allowed to equilibriate into the ground state. We then turn on the magnetic field which is aligned with respect to the rotation axis and the angular momentum of the system. With an increasing magnetic field the ground state of the system evolves towards the ground state of the scalar condensate in $m=1$ or $m=-1$ component. Which component is favored depends on the direction of the magnetic field. As the rotation frequency is small, this ground state has a vanishing angular momentum. Therefore angular momentum of the ground state decreases with increasing magnetic field.

The reverse process of starting with a large magnetic field along the $z$-direction and then turning it off behaves differently. The ground state of the scalar GP equation is metastable under such change. Initially with a large magnetic field the ground state is essentially a scalar condensate without angular momentum. It is hard to relax such a state into a coreless vortex state with finite angular momentum. This behavior is reminescent of the hysteresis for the vortex nucleation in scalar condensates.

\section{CREATION}

As it was already mentioned, the behaviour of the system may be different if the external magnetic field has other non-zero components than the one pointing in $z$ direction. This is dramatically shown in the topological vortex formation [24 27] which was recently demonstrated experimentally [28]. In this experiment a magnetic field configuration of the Ioffe-Pritchard trap was changed in time. In particular the $z$-component of the magnetic field was inverted. Once the $z$-component is inverted, the condensate wavefunction has acquired a winding number 2 . It should be noted that this process continuously transforms a condensate with vanishing angular momenta into a condensate with an angular momentum 2 per particle. When the spreading of the wavefunctions can be ignored this process is equivalent with coreless vortex transformations. In this respect coreless vortices have already been experimentally created. In a IoffePritchard magnetic trap a coreless vortex is not stable since all hyperfine states are not trapped. The stabilization of the coreless vortex structures would require an optical trap.

\section{CONCLUSIONS}

We have studied the ground state properties of rotating spin-1 condensates. We have found out that for a sufficiently small rotating frequency the ground state of a ferromagnetic spinor condensate is a coreless vortex. However, because of the finite size of a condensate a certain critical rotation frequency has to be exceeded for a coreless vortex to appear. We have also investigated antiferromagnetic condensates which in contrast to coreless vortices have rotating ground states exhibiting the discrete staircase behavior.

All the simulations presented in this paper have been performed in two dimensions. Consequently they are expected to describe well pancake shaped condensates. We have also performed simulations with full threedimensional Gross-Pitaevskii equations and convinced ourselves that our results are not specific to two dimensions. While a detailed study in three dimensions is at present too time-consuming, it does seem that our results apply qualitatively even in a cigar shaped trap.

In this paper we have focused solely on the ground state. Naturally the ground state is one thing, but getting the system into it in a reasonable time is quite another. The energy landscape of the rotating spinor condensate is complex and metastable states abound. This may cause problems in the experiments as the relaxation time to the true ground state can become very long. Also the problem of nucleation of coreless vortices may cause problems, especially if the system is initially prepared as a pure stationary condensate. Nevertheless with some effort all these complications can be overcome, and the ground state of the rotating spinor condensate promises to be an exciting field of research. 


\section{ACKNOWLEDGEMENTS}

Authors acknowledge the Academy of Finland (project 50314) for financial support. In addition J.-P. M. was supported by the Stichting voor Fundamenteel Onderzoek der Materie (FOM), which is supported by the Nederlandse Organisatie voor Wetenschaplijk Onderzoek (NWO). A. C. thanks the Magnus Ehrnrooth foundation for financial support.

[1] P. Nozieres and D. Pines, The Theory of Quantum Liquids (Addison-Wesley, Redwood City, CA, 1990).

[2] K. W. Madison, F. Chevy, W. Wohlleben, and J. Dalibard, Phys. Rev. Lett. 84, 806 (2000).

[3] J. R. Abo-Shaeer, C. Raman, J. M. Vogels, and W. Ketterle, Science 292, 476 (2001).

[4] D. A. Butts and D. S. Rokshar, Nature (London) 397, 327 (1999).

[5] D. L. Feder, C. W. Clark, and B. I. Schneider, Phys. Rev. Lett. 82, 4956 (1999).

[6] Y. Castin and R. Dum, Eur. Phys. J. D 7, 399 (1999).

[7] E. Lundh, C. J. Pethick, and H. Smith, Phys. Rev. A 55, 2126 (1997).

[8] D. M. Stamper-Kurn, M. R. Andrews, A. P. Chikkatur, S. Inouye, H.-J. Miesner, J. Stenger, and W. Ketterle, Phys. Rev. Lett. 80, 2027 (1998).

[9] M. D. Barrett, J. A. Sauer, and M. S. Chapman, Phys. Rev. Lett. 87, 010404 (2001).

[10] T.-L. Ho and V. B. Shenoy, Phys. Rev. Lett. 77, 2595 (1996).

[11] N. D. Mermin and T.-L. Ho, Phys. Rev. Lett. 36, 594 (1978).

[12] T. Mizushima, K. Machida, and T. Kita, Phys. Rev. Lett. 89, 030401 (2002).

[13] S.-K. Yip, Phys. Rev. Lett. 83, 4677 (1999).

[14] T.-L. Ho, Phys. Rev. Lett. 81, 742 (1998).

[15] H. Pu, C. K. Law, S. Raghavan, J. H. Eberly, and N. P. Bigelow, Phys. Rev. A 60, 1463 (1999).

[16] E. J. Mueller and T.-L. Ho, Phys. Rev. Lett. 88, 180403 (2002).

[17] P. W. Anderson and G. Toulouse, Phys. Rev. Lett. 38, 508 (1977).

[18] M. M. Salomaa and G. E. Volovik, Rev. Mod. Phys. 59, 533 (1987).

[19] In principle a position dependent $\beta$ will cause an effective potential, proportional to $|\nabla \beta(r)|^{2}$, for the condensate. As will be seen shortly, the function $\beta(r)$ is nearly linear in the high density regions. Consequently such effective potential terms can be safely ignored for reasonable parameter values.

[20] For the corresponding scalar condensate, the critical frequency for the stabilization of a single vortex is about 0.16 .

[21] V. M. Prez-Garca and J. J. Garca-Ripoll, Phys. Rev. A
62, 033601 (2000).

[22] U. Leonhardt and G. E. Volovik, cond-mat/0003428 (2000).

[23] T.-L. Ho and S. K. Yip, Phys. Rev. Lett. 84, 4031 (2000).

[24] M. Nakahara, T. Isoshima, K. Machida, S.-I. Ogawa, and T. Ohmi, Physica B 284-288, 17 (2000).

[25] T. Isoshima, M. Nakahara, T. Ohmi, and K. Machida, Phys. Rev. A 61, 063610 (2000).

[26] S.-I. Ogawa, M. Möttönen, M. Nakahara, T. Ohmi, and H. Shimada, cond-mat/0202018 (2002).

[27] M. Möttönen, N. Matsumoto, M. Nakahara, and T. Ohmi, cond-mat/0205542 (2002).

[28] A. E. Leanhardt, A. Görlitz, A. P. Chikkatur, D. Kielpinski, Y. Shin, D. E. Pritchard, and W. Ketterle, condmat/0206303 (2002). 
This figure "Fig4a.jpg" is available in "jpg" format from: http://arxiv.org/ps/cond-mat/0208078v2 
This figure "Fig4b.jpg" is available in "jpg" format from: http://arxiv.org/ps/cond-mat/0208078v2 
This figure "Fig4c.jpg" is available in "jpg" format from: http://arxiv.org/ps/cond-mat/0208078v2 